\title{
Molecular Characterization and Antimicrobial Susceptibility of Biofilm-forming Acinetobacter baumannii Clinical Isolates from Daejeon, Korea
}

Ji Youn Sung

Department of Biomedical Laboratory Science, Far East University, Eumseong, Korea

\section{대전지역에서 분리된 생물막 형성 Acinetobacter baumannii 임상분리주의 분자유전학적 특성과 항균제 감수성양상}

성지연

극동대학교 임상병리학과

\begin{abstract}
The emergence and dissemination of multidrug-resistant (MDR) Acinetobacter baumannii isolates have been reported worldwide, with most of these possessing the ability to form biofilms. Biofilm formation is an important virulence factor associated with the resistance to disinfection and desiccation. This study examined the genetic basis of antimicrobial resistance mechanisms of biofilm-forming $A$. baumannii clinical isolates. Imaging and quantification of biofilms were performed by a crystal violet assay and 46 biofilm-forming $A$. baumannii isolates were selected. Subsequently, 16 isolates belonging to different clones were identified using REP-PCR, and detection of the antimicrobial determinants in the isolates was carried out. The 16 isolates included 9 non-MDR and 7 MDR isolates. The mean biomass OD $_{560}$ values of the non-MDR (0.96) and MDR (1.05) isolates differed but this difference was not significant. In this study, most biofilm-forming MDR $A$. baumannii isolates contained various antimicrobial resistance determinants (blaxa-23, armA, and mutations of gyrA and parC). On the other hand, most biofilm-forming non-MDR $A$. baumannii isolates did not contain antimicrobial resistance determinants. These results suggest that there is little correlation between the biofilm-forming ability and antimicrobial susceptibility in $A$. baumanniiisolates. In addition, the emergence of MDR A. baumannii clinical isolates is generally caused by mutations of the genes associated with antimicrobial resistance and/or the acquisition of various antimicrobial resistance determinants.
\end{abstract}

Key words: Acinetobacter baumannii, Antimicrobial resistance, Biofilm, Crystal violet assay

This is an Open Access article distributed under the terms of the Creative Commons Attribution Non-Commercial License (http://creativecommons.org/licenses/by-nc/4.0) which permits unrestricted non-commercial use, distribution, and reproduction in any medium, provided the original work is properly cited.

Copyright ( 12018 The Korean Society for Clinical Laboratory Science. All rights reserved.
Corresponding author: Ji Youn Sung Department of Biomedical Laboratory Science, Far East University, 76-32 Daehak-gil,

Gamgok-myeon, Eumseong 27601, Korea Tel: 82-43-879-3668 Fax: 82-43-880-3876

E-mail: azaza72@naver.com

Received: February 14, 2018 Revised $1^{\text {st. }}$ : February 27,2018 Revised 2 ${ }^{\text {nd }}$ : March 13, 2018 Accepted: March 13, 2018

\section{INTRODUCTION}

Acinetobacter baumannii is an important opportunistic and hospital-acquired pathogen responsible for various types of serious infections, such as epidemic pneumonia, secondary meningitis, urinary tract infections, and bacteremia [1]. Many A. baumannii clinical isolates causing nosocomial infections exhibit antimicrobial resistance to most major classes of antimicrobial agents, including aminoglycosides, $\beta$-lactams, and fluoro- 
quinolones. Accordingly, infectious diseases caused by $A$. baumannii are often difficult to treat and have been cured with carbapenems, which have been the drugs of choice until recently [2]. However, the emergence of carbapenem-resistant $A$. baumannii isolates has been reported worldwide, with most of these isolates possessing carbapenem-hydrolyzing $\beta$-lactamase genes. Among carbapenemases spread throughout $A$. baumanniii isolates, carbapenem-hydrolyzing class D $\beta$-lactamases (CHDLs) are the most common and have become a current threat, while metallo- $\beta$-lactamase (MBL)-producing A. baumannii isolates have also continued to be identified [3]. Antimicrobial agents belonging to the aminoglycosides and quinolones have also long been used to treat Acinetobacter infections. Subsequently, the emergence of A. baumannii isolates resistant to these agents is not new, and many studies have reported on the resistance mechanisms of these isolates. Resistance to aminoglycosides is most commonly mediated by aminoglycosidemodifying enzymes, but high-level resistance to clinically important aminoglycosides, including amikacin, gentamicin, and tobramycin, results from the acquisition of $16 \mathrm{~S}$ rRNA methyltransferase genes. More recently, a total of $816 \mathrm{~S}$ rRNA methyltransferase enzymes (ArmA, RmtA, RmtB, RmtC, RmtD, RmtE, RmtF, and NpmA) have been identified among pathogenic bacteria, including $A$. baumannii, worldwide [4, 5]. In addition, quinolone resistance among A. baumannil isolates is due to chromosomal mutations in genes located in the quinolone resistance-determining region (QRDR) and/or acquisition of plasmid-mediated quinolone resistance (PMQR) genes. In particular, high-level resistance to quinolones among $A$. baumannii isolates is caused by mutations in the gyrA gene, encoding DNA gyrase $\mathrm{A}$, and/or the parC gene, encoding the topoisomerase IV A subunit, located in the QRDR [6,7]. The development of multidrug-resistant (MDR) A. baumannii isolates has occurred by chromosomal mutation and/or acquisition of antimicrobial resistance genes. However, some recent studies have suggested that biofilms may play a significant role in the antimicrobial resistance of MDR $A$. baumannii isolates [8]. Biofilms allow microorganisms to persist within unfavorable environments and to disseminate under favorable conditions. In addition, biofilms also function as a site for the exchange and spread of antimicrobial resistance determinants. Therefore, the biofilm formation ability of $A$. baumannii isolates enables the colonization of surfaces such as those of hospital instruments, indwelling catheters, and endotracheal tubes $[9,10]$. According to previous studies, the efflux pumps involved in the MDR phenotype play an important role in transporting quorum sensing (QS) molecules out of the cells, and QS molecules are associated with biofilm formation in A. baumannii isolates [11]. Bacterial components playing a significant role in the maturation and maintenance of biofilms have been previously characterized in A. baumannii isolates by researchers. Biofilm-related virulence determinants include outer membrane protein $\mathrm{A}$ (OmpA), the chaperone-usher assembly system of pili (CsuA BABCDE), the A. baumannii biofilm-associated protein (Bap), and PER-1 belonging to the $\beta$-lactamase family [10].

Previous studies have reported that biofilms are significantly involved in chronic $A$. baumannii infections as well as colonization and persistence of the isolates. In addition, the biofilm formation rate among $A$. baumannii isolates is higher $(80 \sim 91 \%)$ than the rate among other Acinetobacter species (5 24\%) [11]. However, few studies of biofilm-forming A. baumannii isolates have been reported from Korea. In this study, we investigated the genetic basis of antimicrobial resistance mechanisms among biofilm-forming $A$. baumannii isolates obtained from Chungcheong, Korea. For selection of genetically diverse strains, clonal selection was performed by repetitive extragenic palindromic (REP)-PCR. We also investigated biofilm-associated genes and assessed the correlation between antimicrobial resistance and biofilm formation among $A$. baumannii isolates.

\section{MATERIALS AND METHODS}

\section{Bacterial isolation and identification}

A total of 58 consecutive and non-duplicate $A$. 
baumannii clinical isolates were collected from a tertiary hospital located in Daejeon, Korea from 2012 to 2016. Until to investigate, the isolates had been stored long term in brain heart infusion broth (Difco Laboratories, Detroit, MI, USA) containing $20 \%$ glycerol at $-70^{\circ} \mathrm{C}$. All A . baumannii isolates were identified using biochemical tests with the VITEK 2 Automated Instrument ID System (bioMérieux, Marcy l'Etoile, France) and analysis of partial rpoB housekeeping gene sequences, as described previously [12].

\section{Detection of biofilm formation}

Biofilm imaging and quantification were performed using the crystal violet assay as described by Martí et al. with some modifications [13]. Each well of a polystyrene-bottomed 96-well plate was filled with $100 \mu \mathrm{L}$ of brain heart infusion broth, in which A. baumannii strains were inoculated at approximately $1 \times 10^{7} \mathrm{CFU} / \mathrm{mL}$. Each of the isolates was inoculated in triplicate. After incubation at $37^{\circ} \mathrm{C}$ for $18 \mathrm{hr}$, planktonic cells from each well were aspirated and well-bound cells were washed three times with sterile deionized water. The biofilm remaining in each well was stained using $1 \%$ crystal violet solution at room temperature for $10 \mathrm{~min}$ and washed three times with sterile deionized water. After the last wash, the biofilm was air-dried at $55^{\circ} \mathrm{C}$ for 30 min. For imaging, biofilms stained with crystal violet were observed by a phase contrast microscope (Meiji Techno, San Jose, CA, USA). Subsequently, stained biofilm cells were dissolved with $100 \mu \mathrm{L}$ of $95 \%$ ethanol, and the optical density (OD) of each well at a wavelength of $560 \mathrm{~nm}$ was measured by a Multiskan GO microplate spectrophotometer (Thermo Fisher Scientific, Langenselbold, Germany). Each assay for isolates involved in this study was performed in triplicate, and averages of triplicate values were calculated. Three $A$. baumannii isolates obtained and confirmed to be nonbiofilm-forming in our previous study were used as negative control strains for biofilm formation [14].

\section{REP-PCR for clonal assessment}

Clonal selection of biofilm-forming A. baumannii isolates was performed using REP-PCR with primers REP1
(5'-IIIGCGCCGICATCAGGC-3') and REP2 (5'-ACGTCTTATCAGGCCTAC-3') [15]. The cycling conditions were as follows: initial denaturation at $95^{\circ} \mathrm{C}$ for $5 \mathrm{~min}$; amplification for 35 cycles of $95^{\circ} \mathrm{C}$ for $1 \mathrm{~min}, 48^{\circ} \mathrm{C}$ for $1 \mathrm{~min}$, and $70^{\circ} \mathrm{C}$ for $6 \mathrm{~min}$; and a final extension at $70^{\circ} \mathrm{C}$ for 10 min. Amplified products were electrophoresed on $2 \%$ agarose gels containing ethidium bromide and visualized with a BioDoc-14 imaging system (UVP, Cambridge, UK). Strains belonging to the same clonal clusters displayed identical REP-PCR profiles with the same band sizes and densities.

\section{Determination of antimicrobial susceptibilities and minimum inhibitory concentrations (MICs)}

The antimicrobial susceptibility of each isolate against 12 antimicrobial agents was analyzed on Müller-Hinton agar (Difco) by the Kirby-Bauer disk diffusion method, according to the CLSI M100-S21 guidelines [3]. The following antimicrobial disks (BBL, Cockeysville, MI, USA) were used: amikacin (30 $\mu \mathrm{g})$, ampicillin/sulbactam (10/10 $\mu \mathrm{g})$, aztreonam $(30 \mu \mathrm{g})$, cefepime (30 $\mu \mathrm{g})$, cefotaxime (30 $\mu \mathrm{g})$, ceftazidime (30 $\mu \mathrm{g})$, ciprofloxacin $(5 \mu \mathrm{g})$, gentamicin $(10 \mu \mathrm{g})$, imipenem $(10 \mu \mathrm{g})$, meropenem $(10 \mu \mathrm{g})$, piperacillin/tazobactam $(100 / 10 \mu \mathrm{g})$, and ticarcillin/clavulanic acid $(75 / 10 \mu \mathrm{g})$. Escherichia coli strain ATCC 25922 was used as a reference strain. Multidrug resistance was defined as resistance to three or more representatives of the following five drug classes: aminoglycosides, carbapenems, cephalosporins, fluoroquinolones, and $\beta$-lactam/ $\beta$-lactam inhibitor [16]. MIC determination against imipenem, meropenem, amikacin, gentamicin, ciprofloxacin and ceftazidime of clonally selected $A$. baumannii isolates was performed with the Epsiolon test (Etest; bioMérieux), according to the guidelines recommended by CLSI M100-S21 [17].

\section{Characterization of biofilm-associated genes and antimicrobial resistance determinants}

Clonally selected $A$. baumannii isolates were subjected to PCR and sequencing assays for detection of biofilmassociated genes and antimicrobial resistance deter- 
minants. Total DNA was extracted from each target strain showing different banding patterns from REP-PCR using a DNA purification kit (SolGent, Daejeon, Korea) according to standardized protocols. PCR amplification was performed in separate $25 \mu \mathrm{L}$ reactions containing $0.7 \mathrm{U}$ Taq DNA polymerase MasterMix (SolGent), 50 ng total
DNA, and 20 pmol of each specific forward and reverse primer (Table 1) [5-7, 18-20]. PCR amplification was performed in a GeneAmp PCR System 9600 thermal cycler (Perkin-Elmer Cetus Corp., Norwalk, CT, USA) with an initial melting of the reaction mixture at $95^{\circ} \mathrm{C}$ for $5 \mathrm{~min}$; followed by 30 cycles of $95^{\circ} \mathrm{C}$ for $30 \mathrm{~s}, 52^{\circ} \mathrm{C}$ for $30 \mathrm{~s}$, and

Table 1. Oligonucleotides used in this study for the detection of biofilm-associated genes and antimicrobial resistance determinants

\begin{tabular}{|c|c|c|c|c|}
\hline Target genes & Primer & Sequence $\left(5^{\prime}-3^{\prime}\right)$ & Gene & Reference \\
\hline \multirow[t]{14}{*}{ Aminoglycoside resistance genes } & $\operatorname{armA}-\mathrm{F}$ & CAAATGGATAAGAATGATGTT & $\operatorname{arm} A$ & [5] \\
\hline & $\operatorname{armA-R}$ & TTATTTCTGAAATCCACT & & \\
\hline & $r m t A-F$ & ATGAGCTTTGACGATGCCCTA & $r m t A$ & [5] \\
\hline & $r m t A-R$ & TCACTTATTCCTTTTTATCATG & & \\
\hline & $r m t B-F$ & ATGAACATCAACGATGCCCT & $r m t B$ & {$[5]$} \\
\hline & $r m t B-R$ & CCTTCTGATTGGCTTATCCA & & \\
\hline & $r m t C-F$ & CGAAGAAGTAACAGCCAAAG & rmtC & [5] \\
\hline & $\mathrm{rmtC}-\mathrm{R}$ & ATCCCAACATCTCTCCСACT & & \\
\hline & $r m t D-F$ & CGGCACGCGATTGGGAAGC & $r m t D$ & {$[5]$} \\
\hline & $r m t D-R$ & CGGAAACGATGCGACGAT & & \\
\hline & $r m t E-F$ & ATGAATATTGATGAAATGGTTGC & $r m t E$ & [5] \\
\hline & $r m t E-R$ & TGATTGATTTCCTCCGTTTTTG & & \\
\hline & rmtF-F & GCGATACAGAAAACCGAAGG & rmtF & [5] \\
\hline & rmtF-R & ACCAGTCGGCATAGTGCTTT & & \\
\hline \multirow[t]{10}{*}{ Qunolone resistance genes } & QnrA-F & AGAGGATTTCTCACGCCAGG & $q n r A$ & {$[6]$} \\
\hline & QnrA-R & TGCCAGGCACAGATCTTGAC & & \\
\hline & QnrB-F & GGMATHGAAATTCGCCACTG & $q n r B$ & {$[6]$} \\
\hline & QnrB-R & TTTGCYGYYCGCCAGTCGAA & & \\
\hline & QnrS-F & GCAAGTTCATTGAACAGGGT & qnrs & [6] \\
\hline & QnrS-R & TCTAAACCGTCGAGTTCGGCG & & \\
\hline & gyrAaF & ATGAGCGTATCGGAAATCC & gyrA & [7] \\
\hline & gyrAaR & ATCAATCCTTCAATCGAGATATTC & & \\
\hline & parCaF & ATGGAAGATAAGCTGACTATG & parc & [7] \\
\hline & parCaR & GTTGGTAAATCCGGAGC & & \\
\hline \multirow[t]{8}{*}{ Biofilm associated genes } & bapF & ATGCCTGAGATACAAATTAT & bap & [19] \\
\hline & bapR & GTCAATCGTAAAGGTAACG & & \\
\hline & ompAF & GTTAAAGGCGACGTAGACG & ompA & [19] \\
\hline & ompAR & CCAGTGTTATCTGTGTGACC & & \\
\hline & per-1F & ATGAATGTCATTATAAAAGC & blapER-1 & [19] \\
\hline & per1R & AATTTGGGCTTAGGGCAGAA & & \\
\hline & cSuEF & CATCTTCTATTTCGGTCCC & cSUE & [19] \\
\hline & csuER & CGGTCTGAGCATTGGTAA & & \\
\hline \multirow[t]{13}{*}{ Carbapenemase genes } & ISAba1 & CCTATCAGGGTTCTGCCTTCT & $\mid S A b a 1$ & [18] \\
\hline & OXA-23-F & GATCGGATTGGAGAACCAGA & blaoxA-23-like & {$[20]$} \\
\hline & OXA-23-R & ATTTCTGACCGCATTTCCAT & & \\
\hline & OXA-24-F & GGTTAGTTGGCCCCCTTAAA & blaoxA-24-like & {$[20]$} \\
\hline & OXA-24-R & AGTTGAGCGAAAAGGGGATT & & \\
\hline & OXA-51-F & TAATGCTTTGATCGGCCTTG & bläoxA-51-like & {$[20]$} \\
\hline & OXA-51-R & TGGATTGCACTTCATCTTGG & & \\
\hline & OXA-58-F & AAGTATTGGGGCTTGTGCTG & blaoxA-58-like & {$[20]$} \\
\hline & OXA-58-R & СCCCTCTGCGCTCTACATAC & & \\
\hline & IMP-F & GGAATAGAGTGGCTTAAYTCTC & bla|MP & {$[20]$} \\
\hline & IMP-R & GGTTTAAYAAAACAACCACC & & \\
\hline & VIM-F & GATGGTGTTTGGTCGCATA & blavim & {$[20]$} \\
\hline & VIM-R & CGAATGCGCAGCACCAG & & \\
\hline
\end{tabular}

Abbreviations: $F$, sense primer; $R$, antisense primer; $H, A / C / T ; M, A / C ; Y, C / T$. 
$72^{\circ} \mathrm{C}$ for $30 \mathrm{~s}$; and a final extension at $72^{\circ} \mathrm{C}$ for $3 \mathrm{~min}$. After confirmation of the presence of appropriate amplified products on $1.5 \%(\mathrm{w} / \mathrm{v})$ agarose gels, the amplicons were purified according to the manufacturer's instructions using a PCR purification kit (SolGent), and these purified amplicons were sequenced using a BigDye terminator cycle sequencing kit (PE Applied Biosystems, Foster City, CA, USA) and an ABI PRISM 3730XL DNA analyzer (PE Applied Biosystems). The DNA sequences of biofilmassociated genes and antimicrobial resistance determinants were confirmed using the NCBI BLAST paired alignment function.

\section{RESULTS}

\section{Biofilm imaging and quantification}

Fifty-eight $A$. baumannii isolates belonging to diverse strains were obtained from various clinical specimens over 5 years. Biofilm imaging and formation assays of all isolates involved in this study and negative control strains were carried out. The mean $\mathrm{OD}_{560}$ value of the negative control strains was 0.27 , and this value was used as a cut-off $\mathrm{OD}$ value for biofilm formation ability. Isolates were classified as follows: $\mathrm{OD}_{560} \leq 0.27$, non-biofilm former; $0.27<\mathrm{OD}_{560}$ $\leq 0.54$, weak biofilm former; $0.54<\mathrm{OD}_{560} \leq 1.08$, moderate biofilm former; $\mathrm{OD}_{560}<0.18$, strong biofilm former. As the $\mathrm{OD}$ value of the biofilm increased, the abundance of matrix-like structures; thick, contained cells; and biofilm-covered surface areas increased (Figure 1). Among the 58 isolates, 12 isolates were non-biofilm formers and $46(79.3 \%)$ were biofilm formers, including 11 (19.0\%), 32 (55.2\%), and $3(5.2 \%)$ isolates categorized as strong, moderate. and weak biofilm formers, respectively.

\section{Association of MDR phenotype with biofilm-forming capability}

Epidemiological typing of the $46 \mathrm{~A}$. baumannii isolates with confirmed biofilm formation capacity was performed by REP-PCR, and a total of 16 REP genotypes were identified (Figure 2). Subsequently, antimicrobial susceptibility test was performed to determine MDR and non-MDR status of 16 randomly selected $A$. baumannii

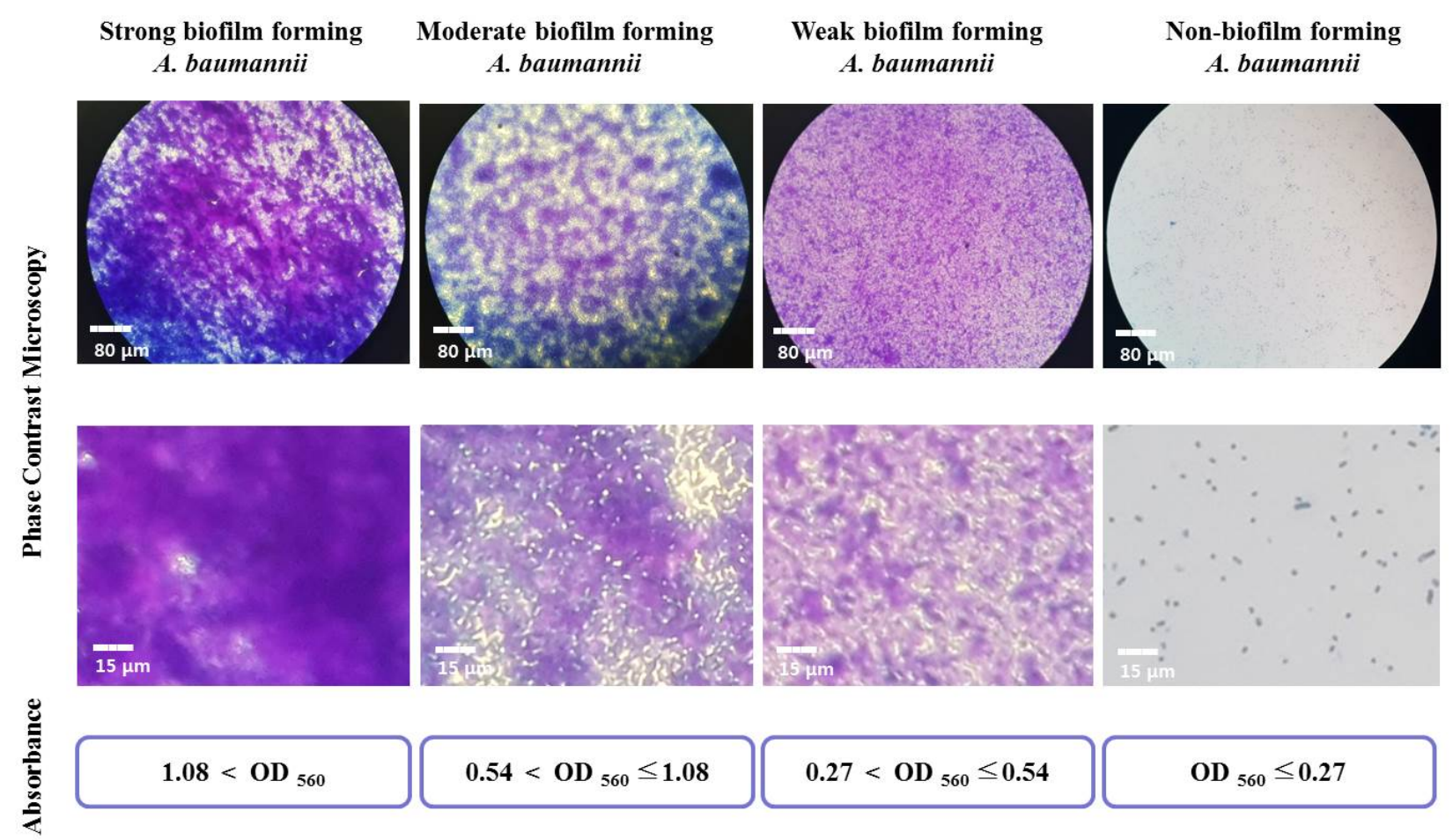

Figure 1. Imaging and quantification of biofilm formation by A. baumannii isolates using a phase contrast microscope and a 96-well microplate spectrophotometer, after crystal violet staining. 
Non-MDR strains

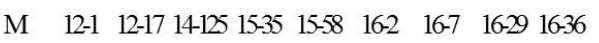

MDR strains

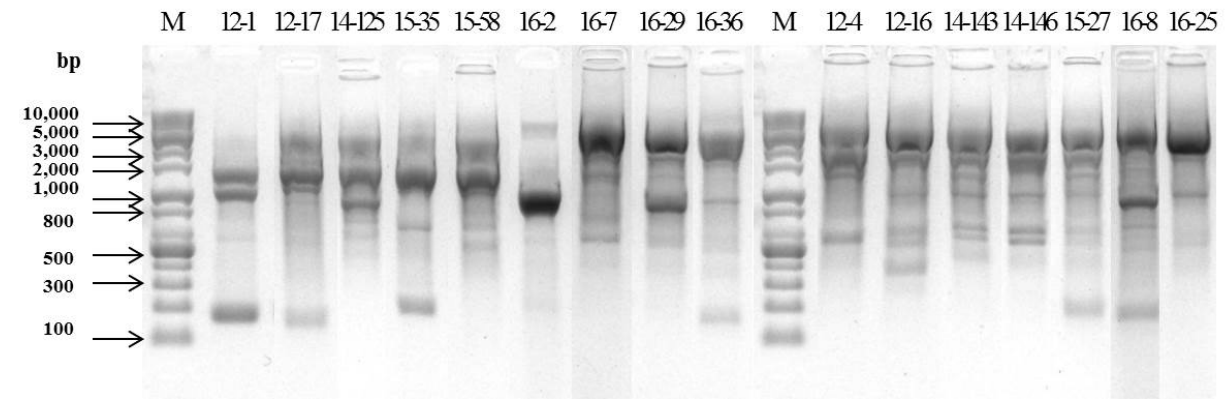

Figure 2. Repetitive extragenic palindromic (REP)-PCR banding pattern of clonal selected nonmultidrug-resistance and multidrug-resistance $A$. baumannii strains, with 1-kb DNA size markers in $\mathrm{M}$ lanes.

Table 2. Antimicrobial susceptibilities of biofilm-forming A. baumannii strains

\begin{tabular}{|c|c|c|c|c|c|c|c|c|c|c|c|c|c|c|c|c|c|c|c|}
\hline \multirow{2}{*}{\multicolumn{2}{|c|}{ Isolates }} & \multirow{2}{*}{ Specimen } & \multicolumn{12}{|c|}{ Antimicrobial susceptibility } & \multirow{2}{*}{$\begin{array}{c}\text { Biomass } \\
\text { OD }_{560^{*}}\end{array}$} & \multicolumn{4}{|c|}{ Biofilm-associated genes } \\
\hline & & & AN & SAM & ATM & FEP & CTX & CAZ & CIP & GM & IPM & MEM & TZP & TIM & & blapER-1 & ompA & CSUE & bap \\
\hline \multirow[t]{9}{*}{ Non-MDR } & $12-1$ & Sputum & $S$ & $S$ & $R$ & $S$ & S & $S$ & $\mathrm{~S}$ & $S$ & $S$ & $S$ & $S$ & S & 1.09 & - & - & + & + \\
\hline & $12-17$ & Sputum & S & $\mathrm{S}$ & $\mathrm{R}$ & $S$ & । & $S$ & $\mathrm{~S}$ & S & $\mathrm{S}$ & S & S & $\mathrm{S}$ & 0.93 & - & + & + & + \\
\hline & $14-125$ & Sputum & S & $\mathrm{S}$ & $\mathrm{R}$ & $\mathrm{S}$ & S & $S$ & $\mathrm{~S}$ & S & $\mathrm{S}$ & S & $S$ & $\mathrm{~S}$ & 0.95 & - & + & + & - \\
\hline & $15-35$ & Bronchial & S & $\mathrm{S}$ & $\mathrm{R}$ & $S$ & I & S & $\mathrm{S}$ & S & $\mathrm{S}$ & S & S & $\mathrm{S}$ & 0.89 & - & + & + & - \\
\hline & $15-58$ & Sputum & S & $\mathrm{S}$ & $\mathrm{R}$ & S & $\mathrm{R}$ & $S$ & $\mathrm{~S}$ & S & $\mathrm{S}$ & S & S & $\mathrm{S}$ & 0.97 & - & + & + & - \\
\hline & $16-2$ & Sputum & S & $\mathrm{S}$ & $\mathrm{R}$ & $S$ & $S$ & S & $\mathrm{S}$ & S & S & S & S & $\mathrm{S}$ & 0.71 & - & - & + & + \\
\hline & $16-7$ & Sputum & S & $\mathrm{S}$ & $R$ & S & $\mathrm{S}$ & S & $\mathrm{S}$ & S & $\mathrm{S}$ & $\mathrm{S}$ & S & $\mathrm{S}$ & 0.89 & - & - & + & + \\
\hline & $16-29$ & Bronchial & $S$ & $S$ & $\mathrm{R}$ & $S$ & 1 & $S$ & $S$ & $S$ & $S$ & $S$ & $S$ & $S$ & 0.89 & - & - & + & + \\
\hline & $16-36$ & Sputum & S & $S$ & $\mathrm{R}$ & $R$ & $R$ & $R$ & $\mathrm{R}$ & S & $\mathrm{S}$ & S & $\mathrm{R}$ & 1 & 1.33 & - & + & + & + \\
\hline \multirow[t]{7}{*}{ MDR } & $12-4$ & Sputum & $\mathrm{R}$ & $\mathrm{R}$ & $\mathrm{R}$ & $\mathrm{R}$ & $\mathrm{R}$ & $R$ & $\mathrm{R}$ & $R$ & R & $\mathrm{R}$ & $\mathrm{R}$ & $\mathrm{R}$ & 1.00 & - & - & - & + \\
\hline & $12-16$ & Sputum & $\mathrm{R}$ & $\mathrm{R}$ & $\mathrm{R}$ & $\mathrm{R}$ & $\mathrm{R}$ & $\mathrm{R}$ & $\mathrm{R}$ & $\mathrm{R}$ & $\mathrm{R}$ & $\mathrm{R}$ & $\mathrm{R}$ & $\mathrm{R}$ & 0.92 & + & + & + & + \\
\hline & $14-143$ & Sputum & $\mathrm{R}$ & $\mathrm{R}$ & $R$ & $\mathrm{R}$ & $\mathrm{R}$ & $R$ & $\mathrm{R}$ & $\mathrm{R}$ & $\mathrm{R}$ & $R$ & $\mathrm{R}$ & $\mathrm{R}$ & 0.91 & - & + & + & - \\
\hline & $14-146$ & Sputum & $\mathrm{R}$ & $\mathrm{R}$ & $\mathrm{R}$ & $\mathrm{R}$ & $\mathrm{R}$ & $\mathrm{R}$ & $\mathrm{R}$ & $\mathrm{R}$ & $\mathrm{R}$ & $\mathrm{R}$ & $\mathrm{R}$ & $\mathrm{R}$ & 0.96 & - & + & + & + \\
\hline & $15-27$ & Sputum & $\mathrm{R}$ & 1 & $R$ & $\mathrm{R}$ & $\mathrm{R}$ & $\mathrm{R}$ & $\mathrm{R}$ & $\mathrm{R}$ & $\mathrm{R}$ & $R$ & $\mathrm{R}$ & $\mathrm{R}$ & 1.70 & - & + & + & + \\
\hline & $16-8$ & Sputum & $R$ & $R$ & $R$ & $\mathrm{R}$ & $\mathrm{R}$ & $R$ & $\mathrm{R}$ & $R$ & $\mathrm{R}$ & $\mathrm{R}$ & $\mathrm{R}$ & $\mathrm{R}$ & 1.04 & - & + & + & + \\
\hline & $16-25$ & Urine & $\mathrm{R}$ & $\mathrm{R}$ & $\mathrm{R}$ & $\mathrm{R}$ & $R$ & $\mathrm{R}$ & $\mathrm{R}$ & $\mathrm{R}$ & $\mathrm{R}$ & $\mathrm{R}$ & $\mathrm{R}$ & $\mathrm{R}$ & 0.80 & - & + & + & + \\
\hline
\end{tabular}

Abbreviations: MDR, multidrug-resistance; AN, amikacin; SAM, ampicillin/sulbactam; ATM, aztreonam; FEP, cefepime; CTX, cefotaxime; CAZ, ceftazidime; CIP, ciprofloxacin; GM, gentamicin; IPM, imipenem; MEM, meropenem; TZP, piperacillin/tazobactam; TIM, ticarcillin/clavulanic acid; S, susceptible; I, intermediate resistant; R, resistant; OD, optical density.

${ }^{*} \mathrm{OD}_{560}$ : Biofilm formation was quantified by measuring optical absorbance $(560 \mathrm{~nm})$ using crystal violet.

isolates, each belonging to a different REP genotype. Among the 16 isolates, 9 and 7 strains were identified as non-MDR and MDR strains, respectively (Table 2). Only one MDR A. baumannii isolate was resistant to 11 antimicrobial agents tested in this study (all agents except ampicillin/sulbactam), while the remaining $6 \mathrm{MDR}$ isolates were resistant to all 12 agents in the panel. In contrast, 8 of the 9 non-MDR A. baumannii isolates were susceptible to 10 antimicrobial agents tested in this study. PCR and sequencing analysis were performed to detect 4 genes associated with biofilm formation in the 16 biofilmforming isolates. Among the 4 genes, $\operatorname{csuE}(93.8 \%)$ was the most prevalent, followed by bap (75.0\%) and ompA
(68.8\%), while the blapER -1 gene (6.3\%) was rare among the isolates (Table 2). Among MDR isolates, the detection rates of ompA (85.7\%) and bap (85.7\%) were higher than the rates of ompA (55.6\%) and bap (66.7\%) among non-MDR isolates. However, the prevalence of the csuE gene was higher among non-MDR isolates (100.0\%) than among MDR isolates (85.7\%). Despite this, there were no significant differences among the detection rates of biofilm-associated genes between MDR and non-MDR isolates. In addition, the mean $\mathrm{OD}_{560}$ values of non-MDR and MDR isolates were 0.96 and 1.05 , respectively, but this difference was not significant (Table 2). 


\section{Correlation between MICs and antimicrobial resistance determinants}

Detection of antimicrobial resistance determinants and MIC determinations for 6 antimicrobial agents were performed to evaluate the association between the presence of these determinants and MDR status (Table 3). All 16 biofilm-forming isolates possessed blaoxa-51-like genes, which are ubiquitous among $A$. baumannii isolates. Among carbapenemase genes, blaoxA-23 and blaoxA-51 were detected in 5 MDR isolates, while only bla0XA-51 was detected in non-MDR isolates. All 5 isolates harboring blaoxA-23 possessed an insertion of ISAba1 upstream of the gene and were resistant to imipenem and meropenem. Among the 16S rRNA methyltransferase genes (armA, $r m t A$, $r m t B$, $r m t C$, $r m t D$, $r m t E$, and $r m t F$ ), only armA was observed in this study. Among the 5 isolates harboring the armA gene, 1 isolate was a non-MDR strain and 4 isolates were MDR strains with high-level resistance to gentamicin
( $>256 \mu \mathrm{g} / \mathrm{mL}$ ). Seven of the 16 isolates possessed point mutations in both gyrA (Ser83Leu) and parC (Ser80Leu or Glu84Lys), and all were resistant to ciprofloxacin with high MICs ( $>32 \mu \mathrm{g} / \mathrm{mL}$ ). However, no isolates involved in this study possessed any qnr genes (qnrA, qnrB, or qnrS).

\section{DISCUSSION}

Among recent studies, many researchers have reported that the ability to form biofilms is an important factor involved in resistance to disinfection and desiccation, as well as antimicrobial agents. In addition, they have reported that biofilms act as a virulence factor, facilitating the extended survival of $A$. baumannii isolates in a hospital environment. The aim of the present study was to analyze the correlation between biofilm-forming ability and antimicrobial resistance in $A$. baumannii isolates. In addition, we evaluated the association between high-level resistance to antimicrobial agents and antimicrobial

Table 3. Characterization of biofilm-forming A. baumannii strains

\begin{tabular}{|c|c|c|c|c|c|c|c|c|c|c|c|c|c|c|}
\hline \multirow{2}{*}{\multicolumn{2}{|c|}{ Isolates }} & \multirow[b]{2}{*}{ Specimen } & \multicolumn{6}{|c|}{ Minimum inhibitory concentrations (MICs) } & \multicolumn{6}{|c|}{ Antimicrobial resistance determinants } \\
\hline & & & MEM & IPM & AMK & $C M$ & CIP & CAZ & $\begin{array}{l}\text { Carbape- } \\
\text { nemase }\end{array}$ & $\begin{array}{l}\text { aba/ } \\
\text { OXA23 }\end{array}$ & $\begin{array}{l}\text { aba/ } \\
\text { OXA51 }\end{array}$ & $\operatorname{armA}$ & gyrA & parC \\
\hline \multirow[t]{9}{*}{ Non-MDR } & $12-1$ & Sputum & 0.25 & 0.38 & 3.00 & 1.50 & 0.13 & 1.50 & OXA-51 & - & + & - & - & - \\
\hline & $12-17$ & Sputum & 2.00 & 1.00 & 8.00 & 4.00 & 0.25 & 2.00 & OXA-51 & - & + & + & - & $\begin{array}{c}80 \text { Ser } \\
\rightarrow \text { Leu }\end{array}$ \\
\hline & $14-125$ & Sputum & 0.25 & 0.38 & 2.00 & 0.50 & 0.13 & 2.00 & OXA-51 & - & - & - & - & - \\
\hline & $15-35$ & Bronchial & 0.38 & 0.38 & 4.00 & 0.75 & 0.19 & 2.00 & OXA-51 & - & - & - & - & - \\
\hline & $15-58$ & Sputum & 1.00 & 0.38 & 3.00 & 1.00 & 0.50 & 3.00 & OXA-51 & - & - & - & - & - \\
\hline & $16-2$ & Sputum & 0.64 & 0.94 & 3.00 & 1.00 & 0.25 & 1.50 & OXA-51 & - & - & - & - & - \\
\hline & $16-7$ & Sputum & 0.23 & 0.47 & 0.75 & 0.25 & 0.25 & 1.00 & OXA-51 & - & - & - & - & - \\
\hline & $16-29$ & Bronchial & 0.47 & 0.64 & 2.00 & 0.25 & 0.38 & 1.50 & OXA-51 & - & - & - & - & - \\
\hline & $16-36$ & Sputum & 0.75 & 1.00 & 3.00 & 6.00 & $>32$ & 48 & OXA-51 & - & - & - & $\begin{array}{l}83 \text { Ser } \\
\rightarrow \text { Leu }\end{array}$ & $\begin{array}{c}80 \text { Ser } \\
\rightarrow \text { Leu }\end{array}$ \\
\hline \multirow[t]{7}{*}{ MDR } & $12-4$ & Sputum & 16 & 16 & 64 & 8 & 4 & 128 & OXA-51 & - & - & - & - & - \\
\hline & $12-16$ & Sputum & $>32$ & $>32$ & 64 & $>256$ & $>32$ & $>256$ & OXA-51 & - & + & - & $\begin{array}{l}83 \text { Ser } \\
\rightarrow \text { Leu }\end{array}$ & $\begin{array}{c}84 \text { Glu } \\
\rightarrow \text { Lys }\end{array}$ \\
\hline & $14-143$ & Sputum & $>32$ & $>32$ & $>256$ & $>256$ & $>32$ & 64 & $\begin{array}{l}\text { OXA-51, } \\
\text { OXA-23 }\end{array}$ & + & - & + & $\begin{array}{l}83 \text { Ser } \\
\rightarrow \text { Leu }\end{array}$ & $\begin{array}{c}80 \text { Ser } \\
\rightarrow \text { Leu }\end{array}$ \\
\hline & $14-146$ & Sputum & 16 & 16 & $>256$ & $>256$ & $>32$ & 128 & $\begin{array}{l}\text { OXA-51, } \\
\text { OXA-23 }\end{array}$ & + & - & + & $\begin{array}{l}83 \text { Ser } \\
\rightarrow \text { Leu }\end{array}$ & $\begin{array}{c}80 \text { Ser } \\
\rightarrow \text { Leu }\end{array}$ \\
\hline & $15-27$ & Sputum & $>32$ & $>32$ & 64 & $>256$ & $>32$ & 128 & $\begin{array}{l}\text { OXA-51, } \\
\text { OXA-23 }\end{array}$ & + & - & - & $\begin{array}{l}83 \text { Ser } \\
\rightarrow \text { Leu }\end{array}$ & $\begin{array}{c}80 \text { Ser } \\
\rightarrow \text { Leu }\end{array}$ \\
\hline & $16-8$ & Sputum & $>32$ & $>32$ & 64 & $>256$ & $>32$ & 64 & $\begin{array}{l}\text { OXA-51, } \\
\text { OXA-23 }\end{array}$ & + & - & + & $\begin{array}{l}83 \text { Ser } \\
\rightarrow \text { Leu }\end{array}$ & $\begin{array}{c}80 \text { Ser } \\
\rightarrow \text { Leu }\end{array}$ \\
\hline & $16-25$ & Urine & 16 & $>32$ & 64 & $>256$ & $>32$ & $>256$ & $\begin{array}{l}\text { OXA-51, } \\
\text { OXA-23 }\end{array}$ & + & - & + & $\begin{array}{l}83 \text { Ser } \\
\rightarrow \text { Leu }\end{array}$ & $\begin{array}{c}80 \text { Ser } \\
\rightarrow \text { Leu }\end{array}$ \\
\hline
\end{tabular}

Abbreviations: MDR, multidrug-resistance; MEM, meropenem; IPM, imipenem; AMK, amikacin; CM, gentamicin; CIP, ciprofloxacin; CAZ, ceftazidime. 
resistance determinants among biofilm-forming $A$. baumannii isolates. For these purposes, we identified a total of $46(79.3 \%)$ biofilm-forming $A$. baumannii isolates, including 11 strong (19.0\%), 32 moderate (55.2\%), and 3 weak (5.2\%) biofilm formers. Our results were similar to previous reports, which showed that more than $75 \%$ of clinical A. baumannii isolates form biofilms [10, 21]. Subsequently, biofilm-forming $A$. baumannii isolates exhibited 16 different REP genotypes and were clonally selected. Biomass analysis results of the 16 isolates, including 9 non-MDR and 7 MDR isolates, were compared. There was little difference between the mean biomass OD560 values of the non-MDR (0.96) and MDR (1.05) isolates. These results suggest that the MDR phenotype is not associated with biofilm-producing ability. Previous researchers have also reported that antimicrobial resistance is similar between biofilm-forming and biofilm non-forming isolates [21]. Next, biofilmassociated genes were investigated in the 16 isolates. We found that MDR isolates had a higher prevalence of biofilm-associated genes, with the exception of csuE, but no significant differences in their biofilm-forming abilities and MDR phenotype were observed based on the presence of biofilm-associated genes. However, one biofilm-associated gene, ompA, was associated with an antimicrobial-resistant phenotype. In our study, only one (9.1\%) of the 11 isolates harboring the ompA gene was susceptible to cefotaxime, while $3(60.0 \%)$ of the 5 isolates not harboring the ompA gene were susceptible to this antibiotic (Table 2). Our results are consistent with previous studies, which reported that OmpA is involved in the attachment and development of biofilms as well as resistance to antimicrobial agents such as cefotaxime, ciprofloxacin, and imipenem [10, 21]. In this study, we investigated correlations between antimicrobial resistance determinants and the MICs of 6 antimicrobial agents. Five (71.4\%) of 7 imipenem- and meropenem-resistant isolates contained the blaoxA-23 gene and the upstream ISAba1 insertion sequence and exhibited the MDR phenotype. These results agree with the worldwide dissemination of the blaoxa-23 gene, which is more prevalent than other carbapenemase genes among MDR A. baumannii isolates [16]. Four (66.7\%) of the 6 amikacin- and gentamicin-resistant isolates harbored armA genes and exhibited high-level resistance to gentamicin ( $>256 \mu \mathrm{g} / \mathrm{mL}$ ). ArmA is widely known as a $16 \mathrm{~S}$ rRNA methyltransferase causing high-level resistance to various antimicrobial agents belonging to the aminoglycosides, and it is most frequently detected among A. baumannii isolates [4]. Quinolone resistance among gram-negative bacteria usually occurs due to chromosomal mutations in QRDRs or the acquisition of PMQR determinants. Seven isolates showed resistance to ciprofloxacin with high MICs $(>32 \mu \mathrm{g} / \mathrm{mL})$ and possessed point mutations in both gyrA and parC. Similarly, other investigators have reported that $A$. baumannii isolates possessing mutations in both gyrA and parC exhibit high-level resistance to ciprofloxacin [7].

In this study, we showed that most biofilm-forming MDR A. baumannii clinical isolates contained various antimicrobial resistance determinants to combat aminoglycosides, carbapenems, cephalosporins, and fluoroquinolones. In addition, most MDR isolates showed high-level resistance to various antimicrobial agents. In contrast, we found that most biofilm-forming non-MDR A. baumannii clinical isolates did not contain antimicrobial resistance determinants and were susceptible to various antimicrobial agents. Therefore, our results suggest that there is little correlation between biofilm-forming ability and antimicrobial susceptibility in MDR $A$. baumannii isolates. However, in this study, there were limitations that the number of clinical isolates was small and the isolates were separated from limited areas. So, further study with a large number of isolates from wide areas might be necessary in future. Correlations between antimicrobial resistance and biofilm-forming ability have been reported in A. baumannii and $P$. aeruginosa isolates [22-24]. Previous researchers demonstrated that $A$. baumannii isolates had achieved high levels of resistance to antimicrobial agents despite producing weak biofilms [22]. They reported that biofilm-forming ability acts as a strategy for $A$. baumannii isolates to get a better survival, particularly in isolates with low levels of resistance to 
antimicrobial agents. However, previous study showed that MDR $P$. aeruginosa isolates produced biofilms at a significantly higher rate than non-MDR isolates [23]. In addition, other study also reported that positive correlations between antimicrobial resistance and biofilm-forming ability in imipenem resistant $A$. baumannii isolates [24]. Therefore, the correlation between biofilm formation and antimicrobial resistance phenotypes remains unclear, and further studies are needed to explore this issue more fully. The present study investigated the genetic basis of antimicrobial resistance mechanisms of biofilm-forming A. baumannii clinical isolates. We confirmed that the emergence of MDR A. baumannii clinical isolates appears to be caused by mutations of genes associated with antimicrobial resistance and/or acquisition of various antimicrobial resistance determinants.

\section{요 약}

다제내성(multidrug-resistant, MDR) Acinetobacter baumannii 균주의 출현 및 확산이 전 세계적으로 보고되어 왔는데, 이들 대부분은 생물막 형성능력을 가지고 있다. 생물막 형성은 소독 및 건조에 대해 세균이 저항할 수 있도록 해주는 중요한 병 독성 인자이다. 본 연구에서는 생물막을 형성하는 A. baumannii 임상 분리주를 대상으로 항균제 내성 기전에 대한 유전 적 기초를 조사하였다. 크리스탈 바이올렛 분석법을 통해 생물 막 형성능력을 확인한 결과 46균주가 생물막을 형성하는 것으 로 나타났다. 이어서 REP-PCR을 수행하여 서로 다른 클론에 속하는 16 균주를 선택한 후 이 균주들을 대상으로 항균제 내성 인자를 검출하였다. 본 연구에서 분리된 9개 non-MDR (0.96) 균주와 7개의 MDR (1.05) 균주의 평균 생물량은 서로 달랐지 만, 이차이는 유의하지 않았다. 대부분의 생물막 형성 MDR 균 주는 다양한 항균제 내성인자 (blaoxA-23, armA 및 $g y r A$ 및 $\operatorname{parc}$ 의 돌연변이)를 가지고 있는 것으로 나타났다. 그러나 대 부분의 non-MDR 균주는 항균제 내성인자를 포함하지 않는 것 으로 나타났다. 우리의 결과는 A. baumannii 균주의 생물막 형 성능력이 항균제 감수성과 상관관계가 적음을 시사한다. 또한 $\mathrm{MDR}$ A. baumannii 임상 분리주의 출현은 대개 항균제 내성과 관련된 유전자의 돌연변이나 또는 다양한 항균제 내성인자의 획득에 의한 것으로 사료된다.

\section{Acknowledgements: None \\ Conflict of interest: None}

\section{REFERENCES}

1. Van Looveren $M$, Goossens $H$, Arpac Steering Group. Antimicrobial resistance of Acinetobacter spp. in Europe. Clin Microbiol Infect. 2004;10:684-704.

2. Richet HM, Mohammed J, McDonald LC, Jarvis WR. Building communication networks: international network for the study and prevention of emerging antimicrobial resistance. Emerg Infect Dis. 2001;7:319-322.

3. Poirel L, Nordmann P. Carbapenem resistance in Acinetobacter baumannii: mechanisms and epidemiology. Clin Microbiol Infect. 2006;12:826-836.

4. Cho YJ, Moon DC, Jin JS, Choi CH, Lee YC, Lee JC. Genetic basis of resistance to aminoglycosides in Acinetobacter spp. and spread of armA in Acinetobacter baumannii sequence group 1 in Korean hospitals. Diagn Microbiol Infect Dis. 2009;64:185190.

5. Hidalgo L, Hopkins KL, Gutierrez B, Ovejero CM, Shukla S, Douthwaite $\mathrm{S}$, et al. Association of the novel aminoglycoside resistance determinant RmtF with NDM carbapenemase in Enterobacteriaceae isolated in India and the UK. J Antimicrob Chemother. 2013;68:1543-1550.

6. Cattoir V, Poirel L, Rotimi V, Soussy CJ, Nordmann P. Multiplex PCR for detection of plasmid-mediated quinolone resistance $q n r$ genes in ESBL-producing enterobacterial isolates. J Antimicrob Chemother. 2007;60:394-397.

7. Hujer KM, Hujer AM, Endimiani A, Thomson JM, Adams MD, Goglin K, et al. Rapid determination of quinolone resistance in Acinetobacterspp. J Clin Microbiol. 2009;47:1436-1442.

8. Rodríguez-Baño J, Martí S, Soto S, Fernandez-Cuenca F, Cisneros JM, Pachon J, et al. Biofilm formation in Acinetobacter baumannii: associated features and clinical implications. Clin Microbiol Infect. 2008;14:276-278.

9. Duarte A, Ferreira S, Almeida S, Domingues FC. Clinical isolates of Acinetobacter baumannii from a Portuguese hospital: PFGE characterization, antibiotic susceptibility and biofilm-forming ability. Comp Immunol Microbiol Infect Dis. 2016;45:29-33.

10. Longo F, Vuotto C, Donelli G. Biofilm formation in Acinetobacter baumannii. New Microbiol. 2014;37:119-127.

11. Varga ZG, Armada A, Cerca P, Amaral L, Mior Ahmad Subki MA, Savka MA, et al. Inhibition of quorum sensing and efflux pump system by trifluoromethyl ketone proton pump inhibitors. In Vivo. 2012;26:277-285.

12. Vijay G, Lenie D, Sophie B, Didier R, Bernard S. Validation of partial $r p o B$ gene sequence analysis for the identification of clinically important and emerging Acinetobacter species. Microbiology. 2009;155: 2333-2341.

13. Martí S, Rodríguez-Baño J, Catel-Ferreira M, Jouenne T, Vila J, Seifert H, et al. Biofilm formation at the solid-liquid and air-liquid interfaces by Acinetobacter species. BMC Res Notes. 2011;11:4-5.

14. Sung JY. Clonal dissemination of multidrug resistant 
Acinetobacter baumannii isolates harboring blaoxA-23 at one university hospital in Daejeon, Korea. Korean J Clin Lab Sci. 2016;48:94-101.

15. Bou G, Cerveró G, Domínguez MA, Quereda C, MartínezBeltrán J. PCR-based DNA fingerprinting (REP-PCR, AP-PCR) and pulsed-field gel electrophoresis characterization of a nosocomial outbreak caused by imipenem- and meropenem-resistant Acinetobacter baumannii. Clin Microbiol Infect. 2000; 6:635-643.

16. Luo TL, Rickard AH, Srinivasan U, Kaye KS, Foxman B. Association of blaоXA-23 and bap with the persistence of Acinetobacter baumannii within a major healthcare system. Front Microbiol. 2015;12:182.

17. Clinical and Laboratory Standards Institute. Performance standards for antimicrobial susceptibility testing; twenty first informational supplement. M100-S21. Wayne, Pensylvania: CLSI, 2011.

18. Mak JK, Kim MJ, Pham J, Tapsall J, White PA. Antibiotic resistance determinants in nosocomial strains of multidrug-resistant Acinetobacter baumannii. J Antimicrob Chemother. 2009;63: 47-54.

19. Omid A, Fereshteh S, Himen S, Farzan M, Mohammad Reza S, Shahla M, et al. Molecular analysis and expression of bap gene in biofilm-forming multi-drug-resistant Acinetobacter baumannii. Rep Biochem Mol Biol. 2016;5:62-72.

20. Woodford N, Ellington MJ, Coelho JM, Turton JF, Ward ME, Brown S, et al. Multiplex PCR for genes encoding prevalent OXA carbapenemases in Acinetobacter spp. Int J Antimicrob Agents. 2006;27:351-353.

21. Thummeepak R, Kongthai P, Leungtongkam U, Sitthisak S. Distribution of virulence genes involved in biofilm formation in multi-drug resistant Acinetobacter baumannii clinical isolates. Int Microbiol. 2016;19:121-129.

22. Lihua Qi, Hao Li, Chuanfu Zhang, Beibei Liang, Jie Li, Ligui Wang, et al. Relationship between antibiotic resistance, biofilm formation, and biofilm-specific resistance in Acinetobacter baumannii. Front Microbiol. 2016;7:483.

23. Abidi SH, Sherwani SK, Siddiqui TR, Bashir A, Kazmi SU. Drug resistance profile and biofilm forming potential of Pseudomonas aeruginosa isolated from contact lenses in Karachi-Pakistan. BMC Ophthalmol. 2013;13:57.

24. Rao RS, Karthika RU, Singh SP, Shashikala P, Kanungo R, Jayachandran S, et al. Correlation between biofilm production and multiple drug resistance in imipenem resistant clinical isolates of Acinetobacter baumannii. Indian J Med Microbiol. 2008;26:333-337. 